MZ-TH/12-25

OUTP-12-13P

\title{
Next-to-leading order QCD corrections to electroweak $Z j j$ production in the POWHEG BOX
}

\author{
Barbara Jäger \\ Institut für Physik (THEP), Johannes-Gutenberg-Universität, 55099 Mainz, Germany \\ E-mail: jaegerba@uni-mainz.de

\section{Steven Schneider} \\ Institut für Physik (THEP), Johannes-Gutenberg-Universität, 55099 Mainz, Germany \\ E-mail: schneste@students.uni-mainz.de \\ Giulia Zanderighi \\ Rudolf Peierls Centre for Theoretical Physics, 1 Keble Road, University of Oxford, UK \\ E-mail: g.zanderighi1@physics.ox.ac.uk
}

\begin{abstract}
We present an implementation of electroweak $Z$-boson production in association with two jets at hadron colliders in the POWHEG framework, a method that allows the interfacing of NLO-QCD calculations with parton-shower Monte Carlo programs. We focus on the leptonic decays of the weak gauge boson, and take photonic and non-resonant contributions to the matrix elements fully into account. We provide results for observables of particular importance for the suppression of QCD backgrounds to vector-boson fusion processes by means of central-jet-veto techniques. While parton-shower effects are small for most observables associated with the two hardest jets, they can be more pronounced for distributions that are employed in central-jet-veto studies.
\end{abstract}

KeYwords: POWHEG, NLO, QCD, SMC. 


\section{Contents}

1. Introduction 1

2. Technical details 2

3. Phenomenological results 6

4. Conclusion 10

\section{Introduction}

One of the most central themes of the CERN Large Hadron Collider (LHC) is the exploration of the mechanism of electroweak symmetry breaking, which in the context of the Standard Model proceeds via a CP-even spin-zero particle, the Higgs boson. The discovery of a new particle that is compatible with the postulated Higgs boson [1] is thus a breakthrough in our understanding of the electroweak interactions. However, to truly establish the existence of a Standard-Model like Higgs boson, besides its mass a variety of additional properties, such as its decay widths, couplings to gauge bosons and fermions, its spin and CP quantum numbers, have to be determined and confronted with theory predictions. This can only be achieved if background processes that are omni-present at hadron colliders are well under control.

A reaction that has already been exploited for the postulated Higgs discovery by the ATLAS [2] and CMS [3] collaborations, and that will play an even more important role in the upcoming determination of the new particle's properties is Higgs production via weak vector boson fusion (VBF), i. e. the purely electroweak $q q \rightarrow q q H$ process that proceeds via weak-boson exchange in the $t$-channel. Because of the color singlet nature of this exchange, VBF processes feature two jets in the forward regions of the detector and little jet activity at central rapidities, while decay products of the Higgs boson tend to be located in between the two tagging jets. These characteristic properties can be exploited to distinguish VBF processes from a priori overwhelming QCD backgrounds.

To explore these features on the basis of a Standard-Model reaction with very similar properties as VBF Higgs production, in Ref. [4] it has been suggested to consider the related process of electroweak $Z j j$ production as a testing ground for the study of $t$-channel color-singlet exchange events. Based on a leading order (LO) parton-level calculation for the electroweak (EW) $q q \rightarrow q q Z$ process, supplemented by explicit matrix elements with an extra parton in the final state for the simulation of additional jet activity, it was shown that color-singlet exchange in the $t$-channel gives rise to soft minijet activity that differs considerably from that of QCD-initiated background processes. In particular, imposing a central 
jet veto (CJV), i. e. discarding events with hard jets at central rapidities, substantially improves the signal significance of VBF processes.

However, in order to reliably predict CJV efficiencies, a precise knowledge of signal and background processes is essential. Within inclusive selection cuts, the dominant source of $Z j j$ events at hadron colliders is QCD-induced production. Next-to-leading order (NLO) QCD corrections to this process are available in the form of a flexible parton-level MonteCarlo program in the public MCFM package $[5,6]$. The virtual corrections of this implementation have been adapted from Ref. [7,8], while the real-emission contributions have first been provided in $[9,10]$. A merging of the NLO-QCD corrections to QCD-induced $Z j j$ production with parton-shower programs has been provided in Ref. [11]. In an inclusive setup, the electroweak $Z j j$ production cross section is much smaller than the QCD induced once. However, once VBF-specific selection cuts are applied, the signature of the electroweak production mode is quite distinct and can thus be considered separately. The NLO-QCD corrections to electroweak $Z j j$ production, including "signal-type" VBF diagrams, but also $Z / \gamma^{\star}$ bremsstrahlung and non-resonant contributions, were computed in Ref. [12]. They are available in the public VBFNLO program package [13]. However, an interface of this code to a parton-shower program (PS) such as HERWIG [14,15] or PYTHIA [16] is not available to date.

In this work, we aim at providing this as yet missing tool by implementing an NLOQCD calculation for electroweak $Z j j$ production in the so-called POWHEG BOX [17], a framework that allows to match dedicated NLO-QCD calculations with public parton-shower programs as described in some detail, e. g. in Refs. $[18,19]$. After an outline of the technical aspects of this endeavor in Sec. 2, we provide phenomenological results in Sec. 3. The theoretical uncertainties associated with our calculation are discussed and the impact of parton-shower effects on observables that are utilized in CJV studies is illustrated. Our conclusions are given in Sec. 4.

\section{Technical details}

For developing an interface between a parton-level calculation for VBF-induced $Z j j$ production at NLO-QCD accuracy and parton-shower programs, we have made use of the publicly available POWHEG BOX [17]. This package contains all process-independent building blocks needed for the matching of a dedicated NLO-QCD calculation in the context of the POWHEG framework $[18,19]$ with multi-purpose parton-shower Monte-Carlo programs. Process-specific ingredients have to be provided by the user of the POWHEG BOX. These include in particular:

- a list of all flavor structures contributing to the Born process,

- the Born phase space,

- the Born amplitudes squared for all partonic subprocesses and the color correlated and the spin correlated Born amplitudes,

- the Born color structure in the limit of a large number of colors, 
- the finite part of the virtual corrections,

- a list of all flavor structures contributing to the real-emission process,

- the real-emission matrix elements squared for all partonic subprocesses.

Once these building blocks have been implemented, the POWHEG BOX itself takes care of infrared singularities by means of an FKS-type subtraction procedure [20].

The user can choose to run the program in a parton-level mode at LO or NLO-QCD, which is particularly useful for validation purposes. In addition, the POWHEG BOX provides the interface to any $p_{T}$-ordered parton-shower program such as PYTHIA. Transverse momentum ordering is essential, since the POWHEG method relies on generating the hardest emission in an event first, while subsequent emissions have to be provided by the partonshower program. The POWHEG BOX can also be matched with angular-ordered parton shower programs, such as HERWIG, if a so-called vetoed-truncated shower is provided. The public version of HERWIG does not offer this option, however, and results obtained by combining an NLO-QCD calculation via the POWHEG BOX with HERWIG can thus only be accurate up to small effects of this missing vetoed-truncated shower. For our phenomenological analysis, we will therefore restrict ourselves to POWHEG matched with PYTHIA.

In the course of the last few years, a variety of Standard-Model processes involving jets in the final state has successfully been implemented in the POWHEG BOX, including dijet production [21] and QCD and EW $H j j[22,23]$ and $W^{+} W^{+} j j[24,25]$ production. Here, we follow closely the procedure of Ref. [25] for the $W^{+} W^{+} j j$ mode with appropriate modifications related to the reduced multiplicity and more involved singularity structure of the electroweak $Z j j$ production process.

Electroweak $Z j j$ production can proceed via processes of the type $q q \rightarrow q q Z$ with color-singlet $\gamma / Z$ or $W$ exchange, referred to as neutral current (NC) and charged current (CC) reactions, respectively. Numerically, the CC contributions dominate over the NC contributions, mainly because of the larger coupling of the quarks to $W$ bosons than to $Z$ bosons and photons. In order to retain all possible angular correlations between leptonic decay products of the $Z$ boson, we consider EW processes of the type $q q^{\prime} \rightarrow \ell^{+} \ell^{-} q q^{\prime}$ (and all related sub-processes with quarks being replaced by anti-quarks), where $\ell^{ \pm}$denotes an $e^{ \pm}$or a $\mu^{ \pm}$. Since the same final state can be produced via a virtual photon rather than a $Z$ boson, diagrams with a $\gamma^{\star}$ have to be taken into account as well. Non-resonant diagrams, where the $\ell^{+} \ell^{-}$pair does not stem from a $Z / \gamma^{\star}$-decay but is produced in the $t$-channel have been included. Some representative Feynman diagrams for the $u s \rightarrow \ell^{+} \ell^{-} d c$ subprocess are depicted in Fig. 1. In all diagrams, we are using a fixed width in the $Z$ - and $W$-boson propagators. The uncertainty related to the treatment of massive gauge-boson propagators is at the order of $0.5 \%$ [12], and can thus be considered as a minor contribution to higher-order electroweak corrections.

Note that we do neglect quark-antiquark annihilation diagrams that contain vectorboson pair production with subsequent decay of one of the weak bosons into a pair of jets, and interference effects between $t$-channel and $u$-channel diagrams in subprocesses with identical quarks. As explained in some detail in Ref. [12], in the phase-space regions where 


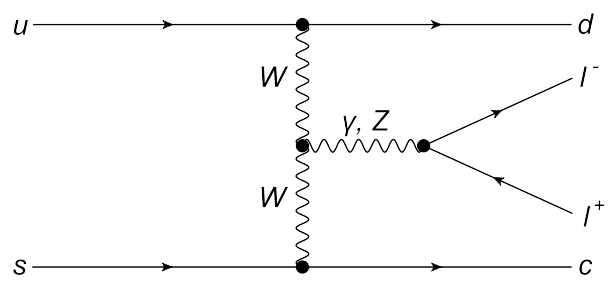

(a)

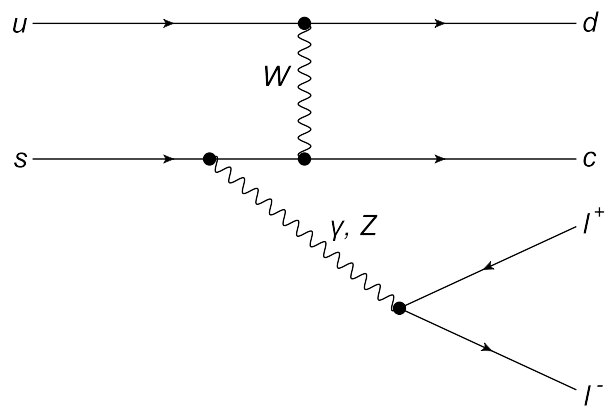

(c)

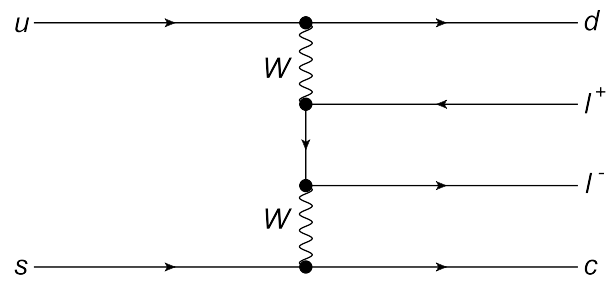

(b)

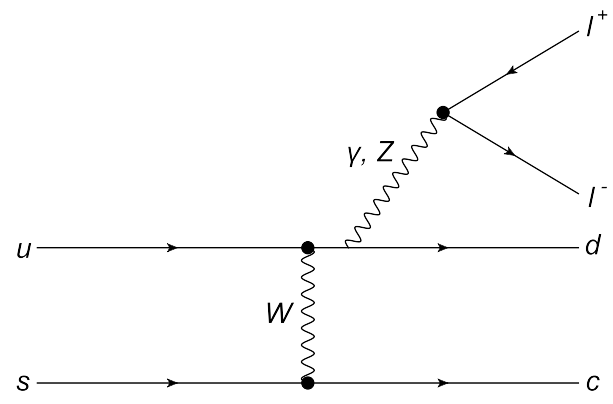

(d)

Figure 1: Sample diagrams for the partonic subprocess $u s \rightarrow \ell^{+} \ell^{-} d c$ at leading order.

VBF processes are searched for, these contributions are entirely negligible, amounting to no more than $0.3 \%$ of the full LO results, once VBF-specific selection cuts are applied. In the absence of selection cuts, at NLO initial-state singularities can arise from collinear $q \rightarrow q g$ and $g \rightarrow q \bar{q}$ splittings. They are taken care of by being factorized into the respective quark and gluon distribution functions of the scattering hadrons. Similar divergences can occur in diagrams where a photon of low virtuality is exchanged in the $t$-channel, thus giving rise to a collinear $q \rightarrow q \gamma$ configuration. Such contributions are considered as part of the QCD corrections to $p \gamma \rightarrow Z j j$ that we are not providing in this work. Following the strategy of Ref. [12], to avoid singular contributions of this type we impose a cut on the virtuality of the photon, $Q_{\gamma, \min }^{2}=4 \mathrm{GeV}^{2}$. Contributions of lower virtuality are suppressed by a strong damping factor. We have checked that by varying the value of $Q_{\gamma, \min }^{2}$ in the range from 0.1 to $9 \mathrm{GeV}^{2}$, the cross section within typical VBF cuts does not change within the statistical error. For simplicity we refer to the EW $p p \rightarrow \ell^{+} \ell^{-} j j$ production process within the above approximations as "VBF $Z j j$ production".

In contrast to Higgs and same-sign gauge boson pair production via VBF, the integrated cross section for single gauge boson production in association with two jets is divergent at leading order, unless dedicated selection cuts are applied. Special care is thus required to avoid singularities in the generation of the underlying Born configuration of our simulation. In order to prevent the population of regions in phase space that are discarded anyway as soon as realistic selection cuts are applied on the generated event sample, a so-called Born-suppression factor has been applied in previous works on $Z$ production processes in association with one or two jets in the framework of POWHEG $[11,26]$. In VBF $Z j j$ production, the singular configurations at Born level can be identified by the 
transverse momenta of the two final-state partons and the invariant mass of the leptons $m_{\ell \ell}$. Singular $\gamma^{\star} \rightarrow \ell^{+} \ell^{-}$configurations are most easily taken care of by a generation cut on the dilepton invariant mass, e. g.

$$
m_{\ell \ell}^{\text {gen }}=30 \mathrm{GeV},
$$

supplemented by a tighter analysis cut on $m_{\ell \ell}$ in our numerical studies. The kinematics of the dilepton system is not affected by hadronic parton-shower effects, making the use of an explicit generation cut on $m_{\ell \ell}$ unproblematic as long as we disallow QED radiation by the parton shower.

In addition, we use a Born-suppression factor $F\left(\Phi_{n}\right)$ that vanishes whenever a singular region of the Born phase space $\Phi_{n}$ is approached. The POWHEG BOX then generates the underlying Born kinematics according to a modified $\bar{B}$ function,

$$
\bar{B}_{\text {supp }}=\bar{B}\left(\Phi_{n}\right) F\left(\Phi_{n}\right) .
$$

Similarly to the prescription of Ref. [11], we set

$$
F\left(\Phi_{n}\right)=\left(\frac{p_{T, 1}^{2}}{p_{T, 1}^{2}+\Lambda_{p_{T}}^{2}}\right)^{n}\left(\frac{p_{T, 2}^{2}}{p_{T, 2}^{2}+\Lambda_{p_{T}}^{2}}\right)^{n},
$$

where the $p_{T, i}$ are the transverse momenta of the two outgoing partons of the underlying Born configuration, and the $\Lambda_{p_{T}}$ and $n$ are technical parameters to be set by the user. In regions that are singular because of the outgoing partons' configuration, the function $F\left(\Phi_{n}\right)$ approaches zero fast enough to yield a finite value for $\bar{B}\left(\Phi_{n}\right) F\left(\Phi_{n}\right)$. The function $\bar{B}_{\text {supp }}$ can therefore be used to generate underlying Born configurations. The generated events then have to be weighted with an extra factor $1 / F\left(\Phi_{n}\right)$ to compensate for the artificial suppression. As default in our analysis we are using the Born-suppression factor of Eq. (2.3) with $\Lambda_{p_{T}}=10 \mathrm{GeV}, n=2 .^{1}$

At NLO-QCD, the interference of the Born amplitudes with one-loop diagrams and real-emission amplitudes squared have to be considered. Within our approximations, the virtual corrections comprise only up to box-type corrections to either the upper or the lower quark line. Diagrams where a gluon is exchanged between the two quark lines vanish when interfered with the Born amplitude because of color conservation. Following the procedure of Ref. [27], the finite parts of the virtual corrections have been calculated numerically by a Passarino-Veltman type tensor reduction. To the real-emission contributions, diagrams with an extra gluon in the final state [such as the subprocess $q q^{\prime} \rightarrow \ell^{+} \ell^{-} q q^{\prime} g$ ] and crossingrelated reactions with a gluon in the initial state [e. g., $g q^{\prime} \rightarrow \ell^{+} \ell^{-} q q^{\prime} \bar{q}$ ] contribute. For the calculation of the respective matrix elements we employ the helicity-amplitude formalism of Ref. [28].

While infrared singularities in the NLO-QCD contributions have been treated by means of a Catani-Seymour dipole subtraction procedure [29] in the parton-level Monte-Carlo program of Ref. [12], the user of the POWHEG BOX does not need to provide subtraction terms

\footnotetext{
${ }^{1}$ Instead of using a Born-suppression factor, one could use suitable generation cuts on the transverse momenta of the final-state partons in the Born configuration.
} 
explicitly. The POWHEG BOX rather generates itself counterterms that take care of potential singularities in soft and collinear configurations in the context of the FKS subtraction procedure [20], using process-specific information contained in the partonic matrix elements. In addition, the program checks automatically that the real-emission contributions approach their soft and collinear limits correctly. While this latter test provides a useful handle to verify the flavor structure and implementation of the real-emission amplitudes by the user, comparing integrated cross sections at NLO-QCD accuracy obtained with the POWHEG BOX in the FKS framework with a stand-alone parton-level code based on the Catani-Seymour dipole subtraction formalism yields a strong check on the entire set-up of the code.

The virtual corrections of our implementation have been checked against the corresponding contributions of Ref. [12] in the public code [13] at amplitude level. In order to validate our tree-level and real-emission amplitudes we have compared them for selected phase-space points to respective amplitudes generated automatically by the MadGraph package $[30,31]$. We found agreement at the level of 10 significant digits. In addition, we have run the POWHEG BOX in a parton-level standalone mode at LO and NLO and compared integrated cross sections as well as a variety of kinematic distributions to an appropriately adapted version of VBFNLO, finding full agreement within the numerical accuracy of the two programs. This provides a strong check on the consistent implementation of all LO and NLO matrix elements as well as on the phase-space integration.

\section{Phenomenological results}

The implementation of VBF $Z j j$ production in the POWHEG BOX is publicly available via the web site of the POWHEG BOX project, http://powhegbox.mib.infn.it, where also instructions for downloading the code are provided. With the downloaded code version, the user can perform studies with her/his own preferred settings. Recommended values for technical parameters and run-time estimates are provided in the documentation of the VBF $Z j j$ code. Here, we wish to present representative results for $p p \rightarrow e^{+} e^{-} j j$ obtained with our POWHEG BOX implementation for VBF-specific settings.

We consider $p p$ collisions at a center-of-mass energy of $\sqrt{s}=8 \mathrm{TeV}$. As electroweak input parameters we use the mass of the $Z$ boson, $m_{Z}=91.188 \mathrm{GeV}$, the mass of the $W$ boson, $m_{W}=80.419 \mathrm{GeV}$, and the Fermi constant, $G_{F}=1.16639 \times 10^{-5} \mathrm{GeV}^{-1}$. The other EW parameters are computed thereof via tree-level electroweak relations. The widths of the weak gauge bosons are set to $\Gamma_{Z}=2.51 \mathrm{GeV}$ and $\Gamma_{W}=2.099 \mathrm{GeV}$, respectively. We assume a diagonal form of the Cabibbo-Kobayashi-Maskawa matrix. For the parton distribution functions of the protons, we use the NLO set of the MSTW2008 parametrization [32] as implemented in the LHAPDF library [33], corresponding to $\alpha_{s}\left(m_{Z}\right)=0.12018$. Contributions with $b$-quarks in the initial state are not taken into account. Jets are defined according to the anti- $k_{T}$ algorithm [34] as available in the FASTJET package [35,36], with $R=0.4$. For our NLO+PS analysis, we will use PYTHIA 6.4.25, including hadronization corrections and underlying event with the Perugia 0 tune. QED radiation effects in the shower are switched off. For the representative results we present below, we set the 
factorization and renormalization scales to

$$
\mu_{F}=\mu_{R}=M_{Z}
$$

Of course, the user is free to make a different choice for the scales when using the code, in particular one could also choose to use dynamical, local scales as suggested in the approach of [37]. For all our analyses we require the presence of at least two jets with

$$
p_{T, j}=20 \mathrm{GeV}, \quad\left|y_{j}\right|<4.5 \text {. }
$$

The two hardest jets inside this rapidity region are referred to as "tagging jets". Furthermore, the invariant mass of the charged lepton pair has to be in a narrow window around the mass of the $Z$ boson,

$$
m_{Z}-10 \mathrm{GeV}<m_{\ell \ell}<m_{Z}+10 \mathrm{GeV},
$$

to avoid contributions from collinear $\gamma^{\star} \rightarrow \ell^{+} \ell^{-}$splittings. In order to enhance the relative importance of $\mathrm{VBF}$ contributions to $p p \rightarrow \ell^{+} \ell^{-} j j$ with respect to potential QCD backgrounds, in addition to Eqs. (3.2) and (3.3) we require the two tagging jets to be well-separated in rapidity, lie in opposite hemispheres of the detector, and exhibit a large invariant mass,

$$
\left|\Delta y_{j_{1} j_{2}}\right|=\left|y_{j 1}-y_{j 2}\right|>4, \quad y_{j 1} \times y_{j 2}<0, \quad m_{j_{1} j_{2}}>600 \mathrm{GeV}
$$

We furthermore require two charged leptons with

$$
p_{T, \ell}>20 \mathrm{GeV}, \quad\left|y_{\ell}\right|<2.5,
$$

well-separated from each other and from the tagging jets,

$$
\Delta R_{\ell \ell}>0.1, \quad \Delta R_{j \ell}>0.4
$$

and in the rapidity range between the two tagging jets,

$$
\min \left\{y_{j_{1}}, y_{j_{2}}\right\}<y_{\ell}<\max \left\{y_{j_{1}}, y_{j_{2}}\right\}
$$

Figure 2 shows our results for the transverse momentum and rapidity distributions of the second hardest tagging jet within the VBF cuts of Eqs. (3.2)-(3.7) at NLO-QCD accuracy and for POWHEG+PYTHIA. The shapes of the NLO curves for these observables do not change considerably when the fixed-order calculation is combined with PYTHIA. The difference between the normalization of the respective NLO and NLO+PS results can be traced back to the increase in the integrated VBF cross section from $\sigma_{\mathrm{NLO}}^{\mathrm{VBF}}=(22.5 \pm 0.2) \mathrm{fb}$ to $\sigma_{\mathrm{NLO}+\mathrm{PS}}^{\mathrm{VBF}}=(23.9 \pm 0.2) \mathrm{fb}$. Similarly, the shapes of distributions related to the first tagging jet or to the two hard leptons are found to remain stable with respect to partonshower effects. This feature helps in identifying VBF processes experimentally, as no contamination of the clean tagging-jet signature is to be expected from parton-shower artifacts. 

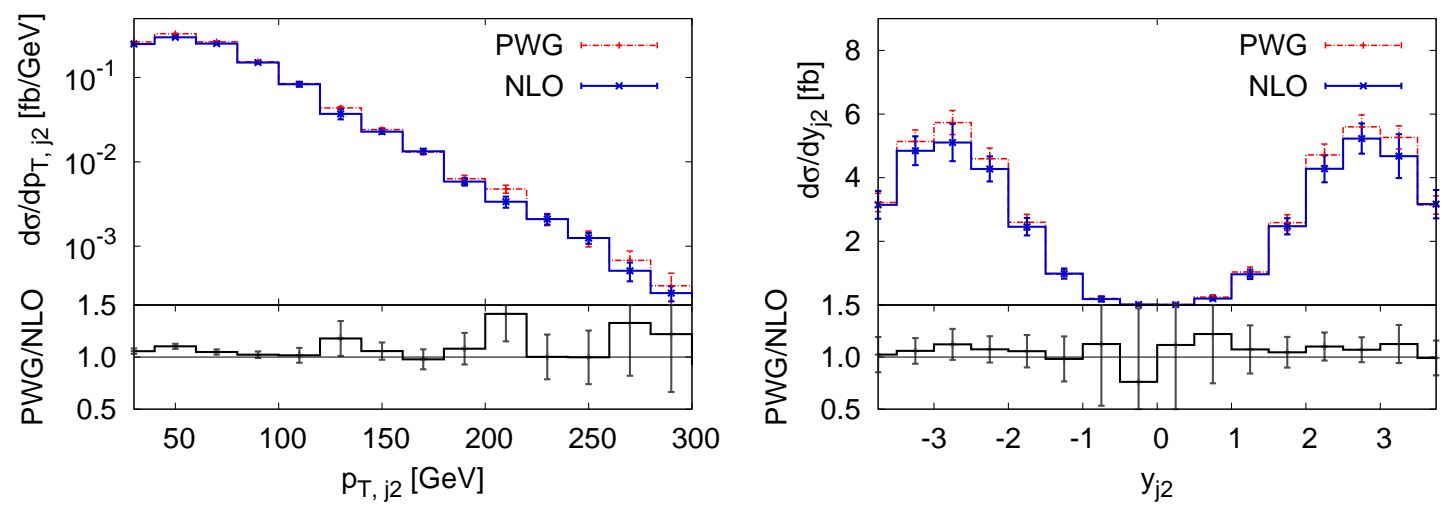

Figure 2: Transverse momentum (left panel) and rapidity distribution (right panel) of the second hardest tagging jet with VBF cuts of Eqs. (3.2)-(3.7) at NLO-QCD ('NLO', solid blue lines) and with POWHEG+PYTHIA ('PWG', dashed red lines)) for $e^{+} e^{-} j j$ production at the LHC with $\sqrt{s}=$ $8 \mathrm{TeV}$. The lower panels show the respective ratios of the POWHEG+PYTHIA to the NLO results.
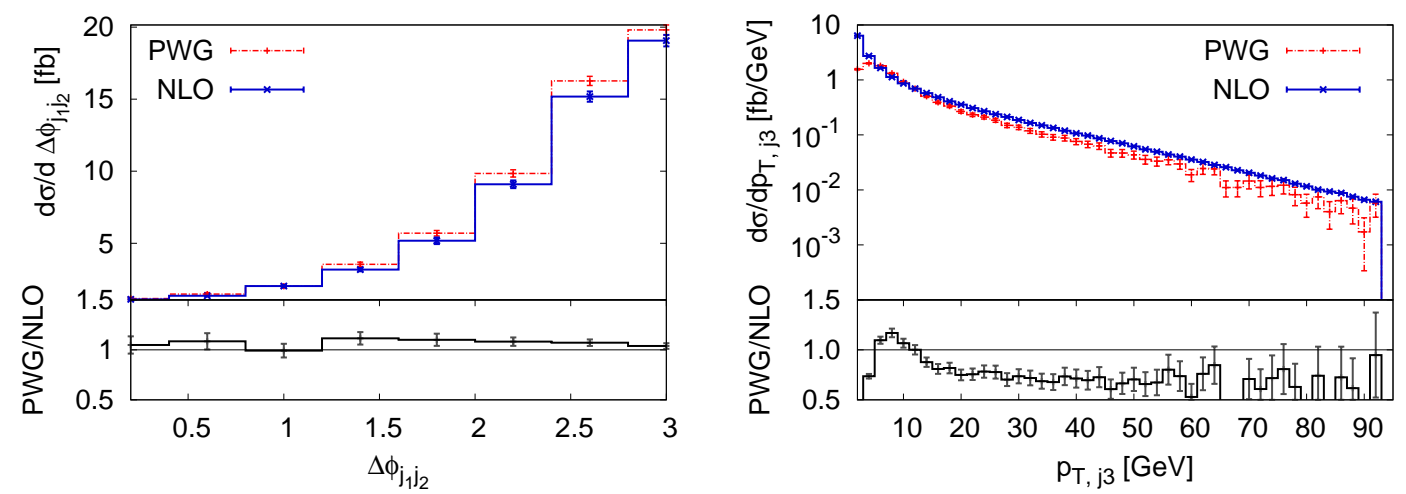

Figure 3: As in Fig. 2 for the azimuthal angle separation of the two tagging jets (left panel) and for the transverse momentum distribution of the third jet with VBF cuts (right panel).

This statement remains true also for correlations between the leptons and the tagging jets, such as the invariant masses of the two hardest jets, of the two hard leptons, or angular correlations such as the azimuthal angle separation of the tagging jets, $\Delta \phi_{j_{1} j_{2}}=\left|\phi_{j_{1}}-\phi_{j_{2}}\right|$. The latter observable is illustrated in Fig. 3 (left), again at NLO and at NLO+PS level.

More pronounced effects of the parton shower are expected for observables that are sensitive to the emission of partons that are not present in the LO configuration of the process under consideration. In a fixed-order perturbative calculation for VBF $Z j j$ production, at NLO QCD a third jet can only stem from the real-emission contributions. When the NLO calculation is merged with PYTHIA, however, extra radiation can also be produced via the parton shower. One therefore expects that the parton shower modifies distributions of the third jet more significantly than those of the hard tagging jets and leptons. Figure 3 (right) demonstrates that, indeed, the transverse momentum distribution of the third jet (i.e., the jet of third-highest $p_{T}$ that is located within the rapidity range of the detector, $\left|y_{j}\right|<4.5$ ) 

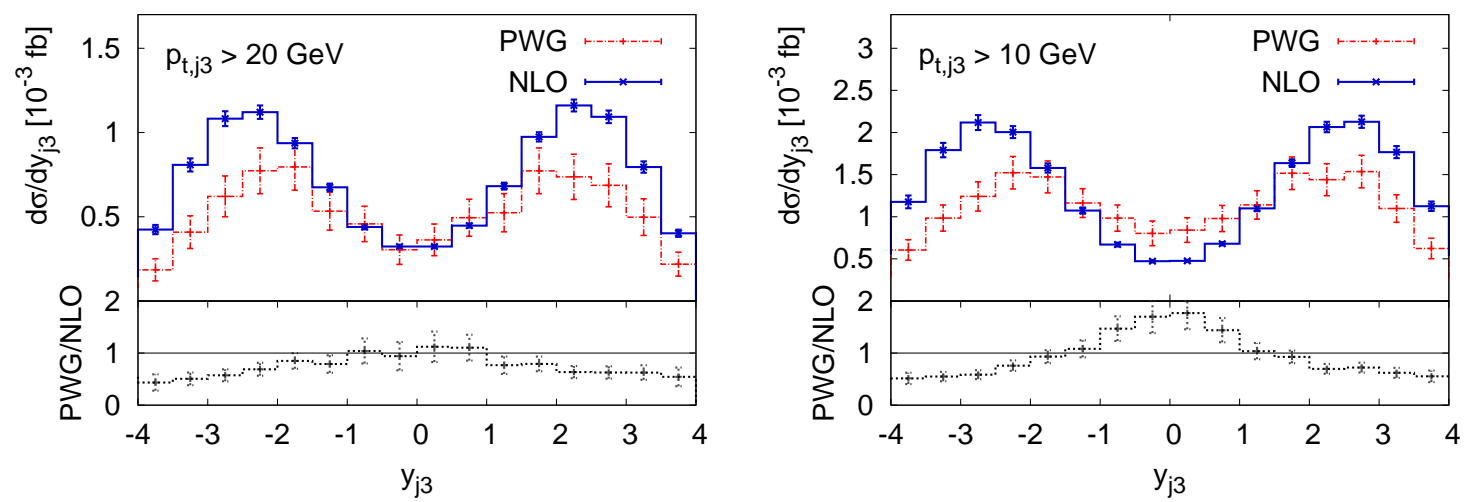

Figure 4: As in Fig. 2 for the rapidity distribution of the third jet with VBF cuts and $p_{T, j_{3}}>$ $20 \mathrm{GeV}$ (left panel) and with $p_{T, j_{3}}>10 \mathrm{GeV}$ (right panel).

changes its shape when the NLO calculation is combined with PYTHIA. While $d \sigma / d p_{T, j_{3}}$ in the fixed-order NLO calculation increases steadily towards small transverse momenta, the rise is damped by the Sudakov form factor in the POWHEG+PYTHIA results.

In order to ease the identification of a jet in an experiment it is typically required to exhibit a transverse momentum larger than $20 \mathrm{GeV}$. Therefore, for the rapidity distribution of the third jet shown in the left panel of Fig. 4 we considered only events with a third jet fulfilling the requirements

$$
p_{T, j_{3}}>20 \mathrm{GeV}, \quad\left|y_{j_{3}}\right|<4.5 \text {. }
$$

Apparently, the parton shower tends to fill the central-rapidity region slightly more than the pure NLO configuration. This effect becomes even more pronounced, if we allow for a softer third jet, weakening the transverse-momentum cut of Eq. (3.8) from 20 to $10 \mathrm{GeV}$, as illustrated by Fig. 4.

An observable particularly suitable for accessing the location of the third jet in rapidity relative to the tagging jets is the variable

$$
y^{\star}=y_{j_{3}}-\frac{y_{j_{1}}+y_{j_{2}}}{2} .
$$

Figure 5 shows that the third jet tends to be located close to one of the tagging jets that are peaked in the rapidity range $\left|y_{j_{1,2}}\right| \approx 2.5 \div 3$ (c.f. Fig. 2), resulting in a maximum of the distribution slightly below $\left|y^{\star}\right| \approx 3$. However, a parton that ends up close to one of the tagging jets is likely to be recombined into this jet, giving rise to a slight dip in $d \sigma / d y^{\star}$ at rapidities related to a maximum in the distribution of a tagging jet. Little radiation occurs in the region in the middle of the two hard jets. If one requires the third jet to fulfill the cuts of Eq. (3.8), the parton-shower does not change this feature significantly. The rapidity gap is filled to some extent, however, by softer jets, as illustrated by the plot on the right-hand-side, where we allow for a third jet of transverse momentum as low as $10 \mathrm{GeV}$, while all other settings remain un-altered. 

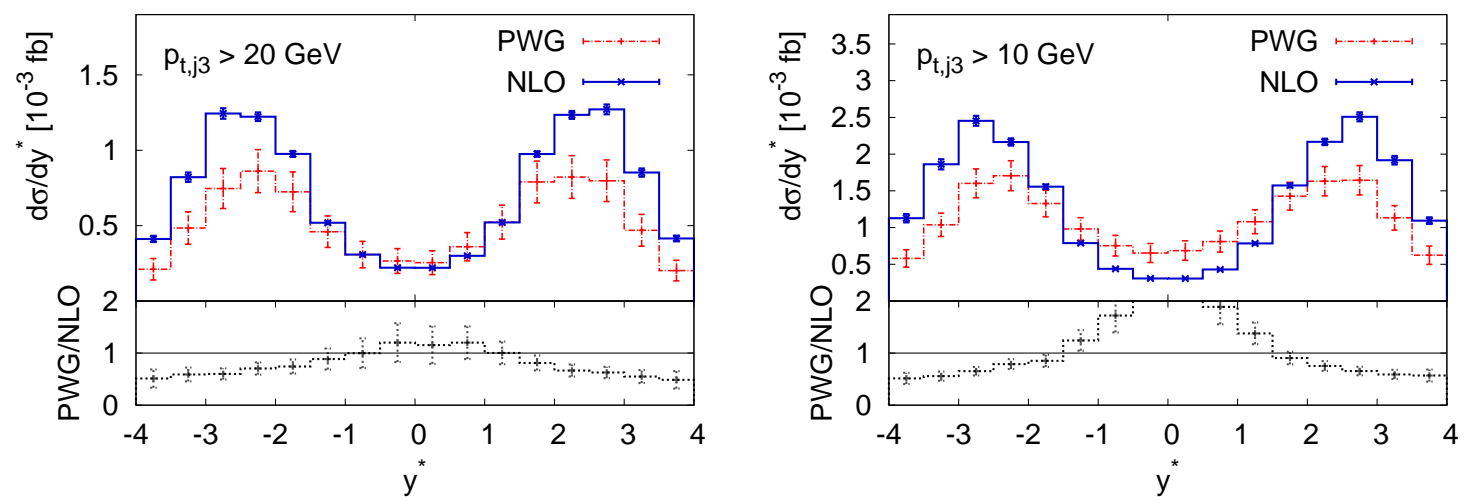

Figure 5: As in Fig. 2 for the rapidity distribution of the third jet with respect to the average of the two tagging jets with VBF cuts and $p_{T, j_{3}}>20 \mathrm{GeV}$ (left panel) and with $p_{T, j_{3}}>10 \mathrm{GeV}$ (right panel).

A further reduction of $\mathrm{QCD}$ backgrounds to the $\mathrm{VBF}$ signal in $Z j j$ final states is expected from a central jet veto, which exploits the unique feature of VBF reactions exhibiting two hard tagging jets that are well separated in rapidity. In addition to the cuts of Eqs. (3.2)-(3.7) we therefore explore the impact of disregarding all events that exhibit at least one extra jet with

$$
p_{T}^{\text {veto }}>20 \mathrm{GeV},
$$

in the rapidity range between the two tagging jets,

$$
\min \left\{y_{j_{1}}, y_{j_{2}}\right\}<y^{\text {veto }}<\max \left\{y_{j_{1}}, y_{j_{2}}\right\}
$$

In case additional jets are emitted they are expected to be located close to the tagging jets rather than in the central-rapidity region. While in a fixed-order parton-level calculation for VBF $Z j j$ production only one extra jet can be produced via the real-emission contributions, in POWHEG+PYTHIA one or more extra jets can be produced via the parton shower. For employing CJV techniques it is essential to understand how such parton-shower effects mitigate the rapidity gap characteristic to VBF reactions. We find that the integrated cross section is reduced by roughly the same modest amount at NLO and NLO+PS level to $\sigma_{\mathrm{NLO}}^{\mathrm{CJV}}=(19.3 \pm 0.2) \mathrm{fb}$ and $\sigma_{\mathrm{NLO}+\mathrm{PS}}^{\mathrm{CJV}}=(21.1 \pm 0.2) \mathrm{fb}$. This behavior is very different from what one expects in the case of QCD-induced $Z j j$ production, c.f. Ref. [11].

\section{Conclusion}

In this work we have presented an implementation of VBF $Z j j$ production in the POWHEG BOX, a framework for merging NLO-QCD calculations with parton-shower programs. We have described the technical details of our implementation, in particular the measures taken to deal with singular regions of the underlying Born configuration. Extensive numerical studies have been performed to verify the independence of phenomenological predictions on technical cuts and reweighting factors. 
We have then presented numerical results for observables that are expected to be utilized in searches for VBF processes at the LHC. Our analysis revealed that the parton shower may change the normalization of cross sections by about 10 to 15 percent, but barely affect the shapes of distributions of the two hardest jets and leptons. Distributions related to additional jet radiation may experience larger changes. However, the benefits of a central-jet veto are hardly diminished by parton-shower effects.

Acknowledgments We are grateful to Paolo Nason for helpful discussions and to Carlo Oleari for a careful reading of the manuscript and valuable comments. The work of B. J. is supported in part by the Research Center Elementary Forces and Mathematical Foundations (EMG) of the Johannes-Gutenberg-Universität Mainz. S. S. acknowledges support from the German Research Foundation (DFG). G. Z. is supported by the British Science and Technology Facilities Council, by the LHCPhenoNet network under the Grant Agreement PITN-GA-2010-264564 and by the European Research and Training Network (RTN) grant Unification in the LHC ERA under the Agreement PITN-GA-2009-237920.

\section{References}

[1] J. Incandela, on behalf of the CMS collaboration; F. Gianotti, on behalf of the ATLAS collaboration, CERN press conference (2012) https://indico.cern. ch/conferenceDisplay . py? conf Id=197461.

[2] R. Hawkings, on behalf of the ATLAS collaboration, ICHEP2012 (2012) https://indico. cern. ch/contributionDisplay py? contribId=4\&conf $I d=181298$.

[3] J. Incandela, on behalf of the CMS collaboration, ICHEP2012 (2012) https://indico. cern. ch/contributionDisplay $\cdot$ py? contribId=5\&conf Id=181298.

[4] D. L. Rainwater, R. Szalapski, D. Zeppenfeld, Phys. Rev. D54 (1996) 6680. [hep-ph/9605444].

[5] J. M. Campbell and R. K. Ellis, Phys. Rev. D 65 (2002) 113007 [hep-ph/0202176].

[6] J. M. Campbell, R. K. Ellis and D. L. Rainwater, Phys. Rev. D 68 (2003) 094021 [hep-ph/0308195].

[7] Z. Bern, L. J. Dixon and D. A. Kosower, Nucl. Phys. B 513 (1998) 3 [hep-ph/9708239].

[8] Z. Nagy and Z. Trocsanyi, Phys. Rev. D 59 (1999) 014020 [Erratum-ibid. D 62 (2000) 099902] [hep-ph/9806317].

[9] F. A. Berends, W. T. Giele and H. Kuijf, Nucl. Phys. B 321 (1989) 39.

[10] K. Hagiwara and D. Zeppenfeld, Nucl. Phys. B 313 (1989) 560.

[11] E. Re, arXiv:1204.5433 [hep-ph].

[12] C. Oleari and D. Zeppenfeld, Phys. Rev. D 69 (2004) 093004. [hep-ph/0310156].

[13] K. Arnold et al., Comp. Phys. Comm. 180 (2009) 1661. [arXiv:0811.4559 [hep-ph]];

K. Arnold et al., arXiv:1107.4038 [hep-ph].

[14] G. Marchesini et al., Comp. Phys. Commun. 67 (1992) 465.

[15] G. Corcella et al., JHEP 0101 (2001) 010. [hep-ph/0011363]. 
[16] T. Sjostrand, S. Mrenna, P. Z. Skands, JHEP 0605 (2006) 026. [hep-ph/0603175].

[17] S. Alioli, P. Nason, C. Oleari, E. Re, JHEP 1006 (2010) 043. [arXiv:1002.2581 [hep-ph]].

[18] P. Nason, JHEP 0411 (2004) 040. [hep-ph/0409146].

[19] S. Frixione, P. Nason, C. Oleari, JHEP 0711 (2007) 070. [arXiv:0709.2092 [hep-ph]].

[20] S. Frixione, Z. Kunszt, A. Signer, Nucl. Phys. B467 (1996) 399. [hep-ph/9512328].

[21] S. Alioli, K. Hamilton, P. Nason, C. Oleari and E. Re, JHEP 1104 (2011) 081 [arXiv:1012.3380 [hep-ph]].

[22] J. M. Campbell, R. K. Ellis, R. Frederix, P. Nason, C. Oleari and C. Williams, arXiv:1202.5475 [hep-ph].

[23] P. Nason, C. Oleari, JHEP 1002 (2010) 037. [arXiv:0911.5299 [hep-ph]].

[24] T. Melia, P. Nason, R. Rontsch and G. Zanderighi, Eur. Phys. J. C 71 (2011) 1670 [arXiv:1102.4846 [hep-ph]].

[25] B. Jäger, G. Zanderighi, JHEP 1111 (2011) 055 [arXiv:1108.0864 [hep-ph]].

[26] S. Alioli, P. Nason, C. Oleari and E. Re, JHEP 1101 (2011) 095 [arXiv:1009.5594 [hep-ph]].

[27] B. Jäger, Phys. Rev. D 81 (2010) 114016 [arXiv:1004.0825 [hep-ph]].

[28] K. Hagiwara and D. Zeppenfeld, Nucl. Phys. B 274 (1986) 1.

[29] S. Catani, M. H. Seymour, Nucl. Phys. B485 (1997) 291. [hep-ph/9605323].

[30] T. Stelzer and W. F. Long, Comput. Phys. Commun. 81 (1994) 357 [hep-ph/9401258].

[31] J. Alwall, P. Demin, S. de Visscher, R. Frederix, M. Herquet, F. Maltoni, T. Plehn and D. L. Rainwater et al., JHEP 0709 (2007) 028 [arXiv:0706.2334 [hep-ph]].

[32] A. D. Martin, W. J. Stirling, R. S. Thorne, G. Watt, Eur. Phys. J. C63 (2009) 189-285. [arXiv:0901.0002 [hep-ph]].

[33] M. R. Whalley, D. Bourilkov, R. C. Group, hep-ph/0508110.

[34] M. Cacciari, G. P. Salam and G. Soyez, JHEP 0804 (2008) 063 [arXiv:0802.1189 [hep-ph]].

[35] M. Cacciari, G. P. Salam, Phys. Lett. B641 (2006) 57. [hep-ph/0512210].

[36] M. Cacciari, G. P. Salam and G. Soyez, arXiv:1111.6097 [hep-ph].

[37] K. Hamilton, P. Nason and G. Zanderighi, arXiv:1206.3572 [hep-ph]. 